\title{
Achievement of Right Ventricular Pacing by Use of a Long Guiding Catheter in a Hemodialysis Patient Presenting Significant Tortuosity of Vasculature
}

\author{
Polychronis Dilaveris, Skevos Sideris, Sofia Vaina, Konstantinos Gatzoulis, \\ and Christodoulos Stefanadis
}

1st University Department of Cardiology, Hippokration Hospital, 15561 Athens, Greece

Correspondence should be addressed to Polychronis Dilaveris, hrodil1@yahoo.com

Received 25 October 2010; Accepted 24 February 2011

Academic Editor: Gavin W. Lambert

Copyright (C) 2011 Polychronis Dilaveris et al. This is an open access article distributed under the Creative Commons Attribution License, which permits unrestricted use, distribution, and reproduction in any medium, provided the original work is properly cited.

\begin{abstract}
Increased vascular calcification and tortuosity are rather common in end-stage renal failure patients who are on hemodialysis. It renders manipulation of catheters and performance of percutaneous transluminal interventions more difficult than expected. Such vascular alterations may be evident in large veins and pose significant difficulties in placement of pacing leads as shown in our case. To overcome such difficulties, we demonstrated in this patient case that long guiding catheters may be of particular value.
\end{abstract}

\section{Introduction}

Cardiovascular disease is the leading cause of death in patients with end-stage renal disease (ESRD). The risk of dying from a cardiovascular event is 10-20 times higher than in the general population, and the prevalence of coronary artery disease in ESRD patients is approximately 40\%. ESRD patients develop cardiovascular abnormalities early in the disease process and have substantial traditional risk factors such as diabetes, hypertension, and dyslipidemia. A feature of many patients in the years preceding a cardiovascular event is the presence of extensive vascular calcification [1]. Increased vascular calcification and tortuosity are rather common in ESRD patients who are on hemodialysis, rendering manipulation of catheters and performance of percutaneous transluminal interventions more difficult than expected. Such vascular alterations may be evident in large veins and pose significant difficulties in placement of pacing leads as shown in our case, herein.

\section{Case Report}

A 76-year-old man who was admitted to hospital with acute inferior infarction underwent primary angioplasty in the culprit right coronary artery. According to his medical history, the patient suffered from an anterior myocardial infarction after which he underwent percutaneous coronary angioplasty in the left anterior descending coronary artery with implantation of two stents. Furthermore, the patient suffered from permanent atrial fibrillation, and he was on chronic hemodialysis program 3 days a week, due to ESRD. During angioplasty the patient developed complete heart block and was supported by a temporary pacing lead in the right ventricle introduced through the femoral vein. Since complete heart block was not relieved three days later, he was referred for implantation of a permanent pacing system. Initially, a right cephalic approach was attempted in order to protect the left arm with the AV shunt. Because the right cephalic vein was heavily calcified and could not sustain the pacing lead or even a guide wire, a right subclavian vein puncture was successfully attempted. Because of the failure to advance the guide wire from the subclavian vein to superior vena cava, venography was performed which revealed complete obstruction of the vein route (Figure 1). Thereafter, a left-sided pectoral implantation was attempted. Since no left-sided cephalic vein was found on incision, a successful subclavian vein puncture was accomplished, and a guide wire was introduced. The guide wire was 


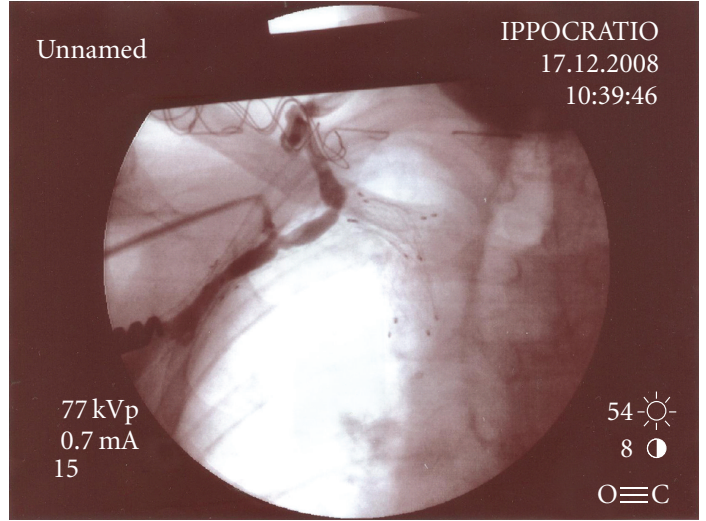

Figure 1: Complete obstruction of the right subclavian vein during venography.

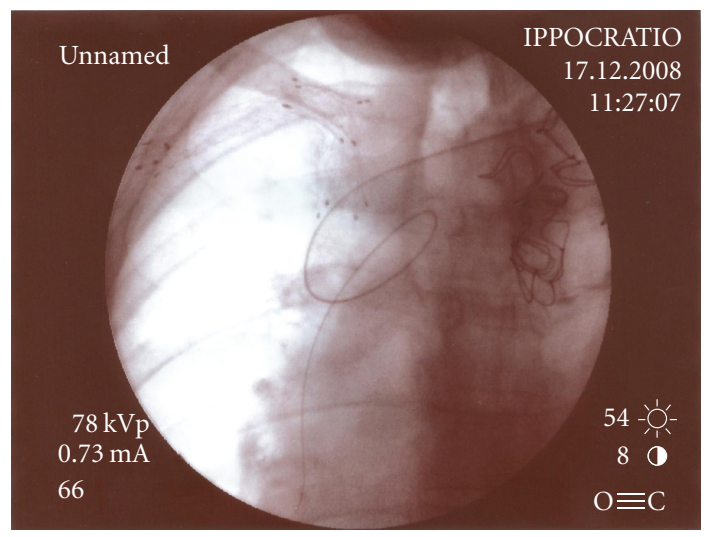

Figure 2: The guide wire is advanced through the left subclavian vein to the right atrium, but with a loop because of significant tortuosity of the superior vena cava.

advanced to the right atrium, but with a loop because of significant tortuosity of the superior vena cava (Figure 2). A 7 Fr. percutaneous introducer was easily advanced over the guide wire into the left subclavian vein, and the guide wire was removed. An active fixation lead (Crystalline ACT FIX ICQ09B Vitatron B.V., Arnhem, The Netherlands, $58 \mathrm{~cm}$ long) was introduced through the subclavian introducer but failed several times to be advanced to the right atrium and formed loops in the superior vena cava area. Therefore, we removed the lead and exchanged the $7 \mathrm{Fr}$. percutaneous introducer with a $9 \mathrm{Fr}$ one. Through the last introducer we advanced a long $(180 \mathrm{~cm})$ hydrophilic guide wire (Radifocus RF GA35183M, Terumo Europe NV, Leuven, Belgium) and over it a long $(45 \mathrm{~cm})$, slittable, guiding catheter (Medtronic Attain LDS 6216A, Medtronic Inc., Minneapolis, Minn, USA) to the right atrium and through the tricuspid valve to the right ventricle (Figure 3 ). Such long guiding catheters are commonly used to cannulate coronary sinus in cardiac resynchronization therapy [2]. Through the long guiding catheter the pacing lead was easily advanced to the right ventricle and screwed in the endocardial wall (Figure 4). Next, the long guiding catheter was slit, the percutaneous

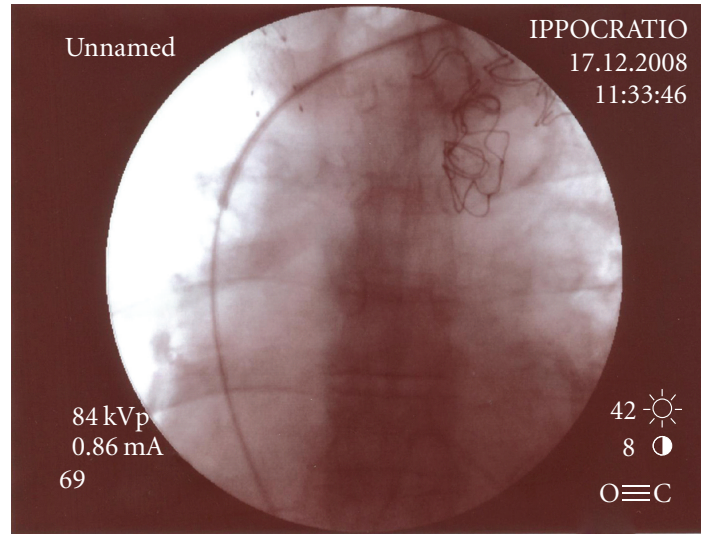

FIGURE 3: A long, slittable, guiding catheter is advanced to the right atrium and through the tricuspid valve to the right ventricle.

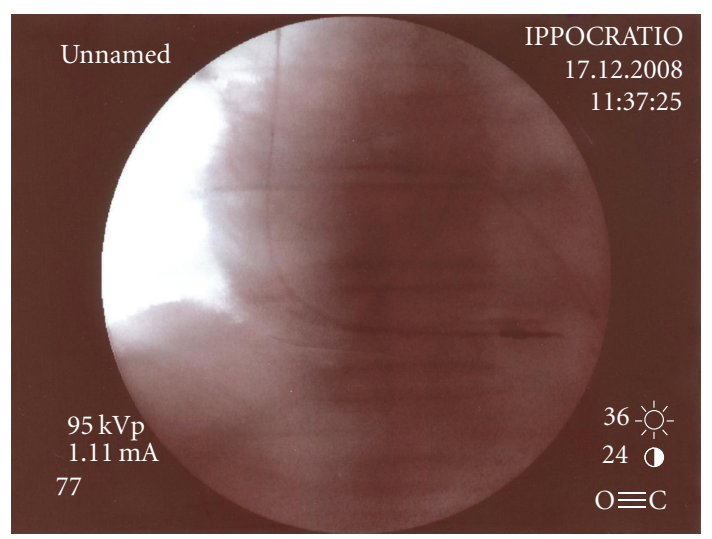

Figure 4: Through the long guiding catheter the pacing lead is advanced to the right ventricle and screwed in the endocardial wall.

introducer removed, and the pulse generator implantation was finished uneventfully. Finally, the temporary pacing lead was removed.

\section{Discussion}

Cardiovascular disease is the leading cause of death in patients with ESRD. Although ESRD patients develop cardiovascular abnormalities early in the disease process and have substantial traditional risk factors such as diabetes, hypertension, and dyslipidaemia, a feature of many patients in the years preceding a cardiovascular event is the presence of extensive vascular calcification [1]. Although the mechanism by which vascular calcifications develop in ESRD patients is complex and is not well known, a possible explanation is that inflammation may contribute to arterial calcification in the presence of an increased serum phosphorus and calcium load [3]. Multiple vascular calcifications and tortuosities are more common in ESRD patients and cause more difficulties in catheters manipulations and/or transluminal interventions [2]. Such vascular alterations may be evident in large veins too and pose significant difficulties in placement of pacing leads as shown in our case. Moreover, 
vascular complications, such as rupture or formation of spurious aneurysms, may occur more often in renal patients. Therefore, the introduction of a $9 \mathrm{Fr}$ guide, as in our case, is more traumatic than a $7 \mathrm{Fr}$ guide, and it carries with it the risk of heavy bleeding, especially in hemodialysis patients who often present coagulation disorders. Consequently, in ESRD patients, a $9 \mathrm{Fr}$ percutaneous introducer and a long guiding catheter should be considered only after failure of the standard procedure. In our ESRD patient, the use of a 9 Fr percutaneous introducer and a long guiding catheter was uncomplicated and proved to be of particular value to accomplish successful placement of a right ventricular pacing lead. However, it needs further investigation in future studies.

\section{References}

[1] H. H. Jung, S. W. Kim, and H. Han, "Inflammation, mineral metabolism and progressive coronary artery calcification in patients on haemodialysis," Nephrology Dialysis Transplantation, vol. 21, no. 7, pp. 1915-1920, 2006.

[2] F. Zanon, E. Baracca, G. Pastore et al., "Implantation of left ventricular leads using a telescopic catheter system," Pacing and Clinical Electrophysiology, vol. 29, no. 11, pp. 1266-1272, 2006.

[3] D. Brancaccio, C. Tetta, M. Gallieni, and V. Panichi, "Inflammation, CRP, calcium overload and a high calcium-phosphate product: a 'liaison dangereuse', Nephrology Dialysis Transplantation, vol. 17, no. 2, pp. 201-203, 2002. 


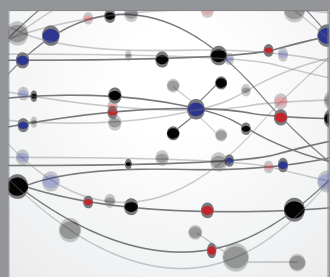

The Scientific World Journal
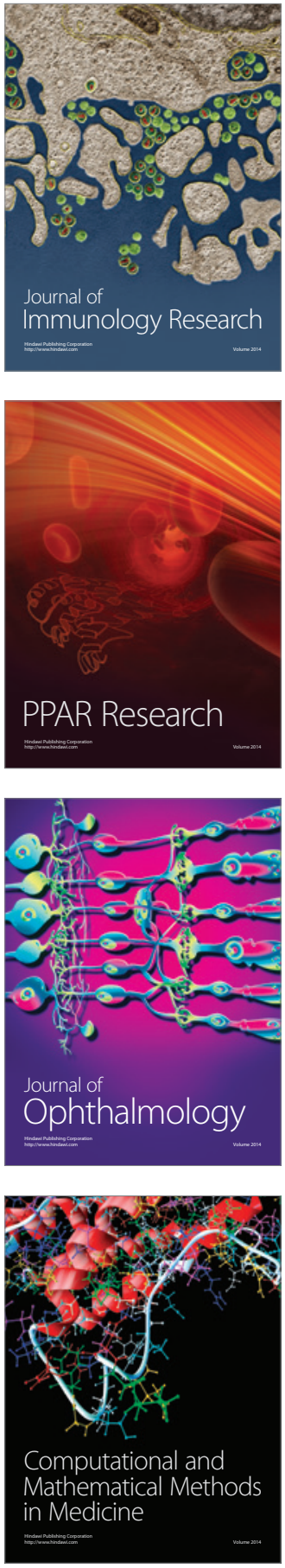

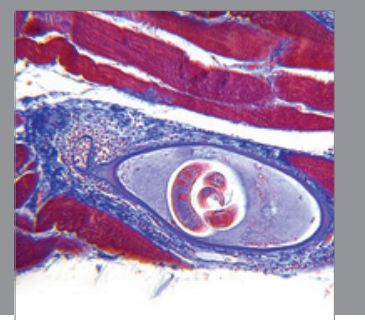

Gastroenterology

Research and Practice
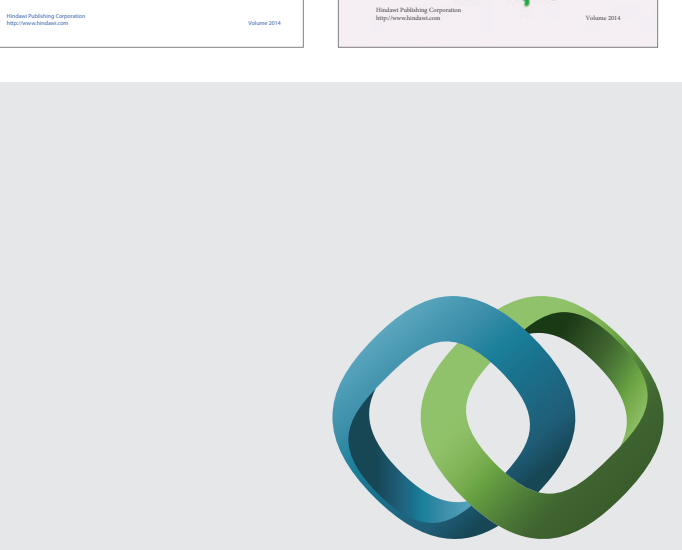

\section{Hindawi}

Submit your manuscripts at

http://www.hindawi.com
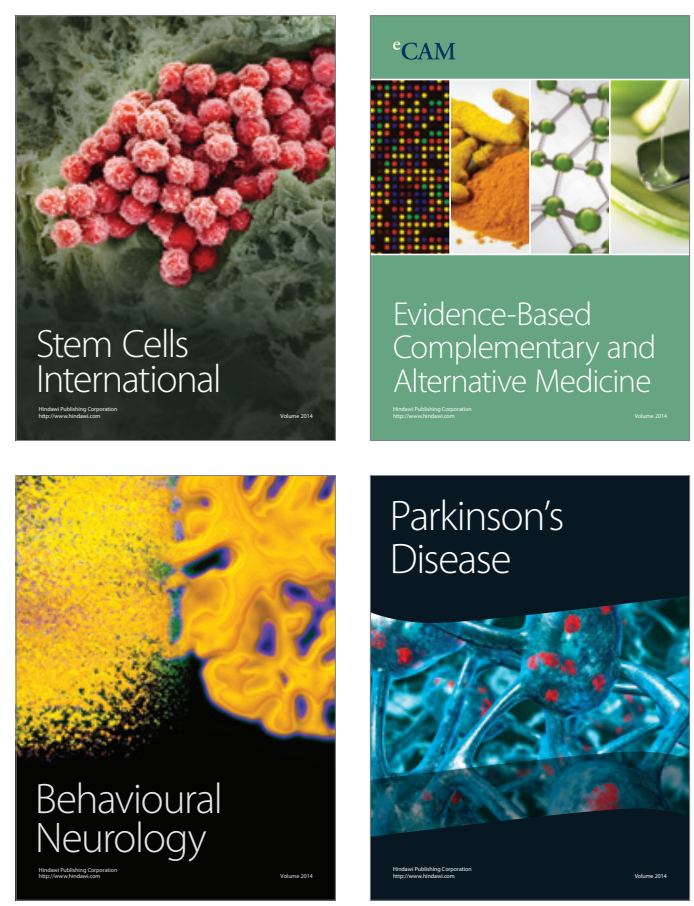

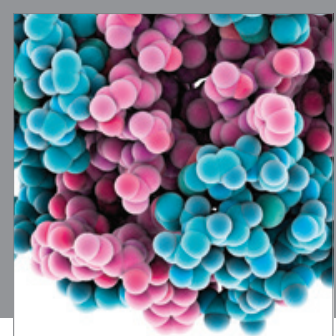

Journal of
Diabetes Research

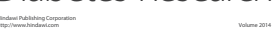

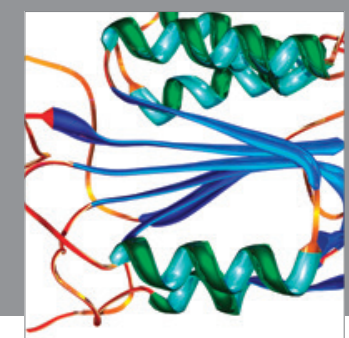

Disease Markers
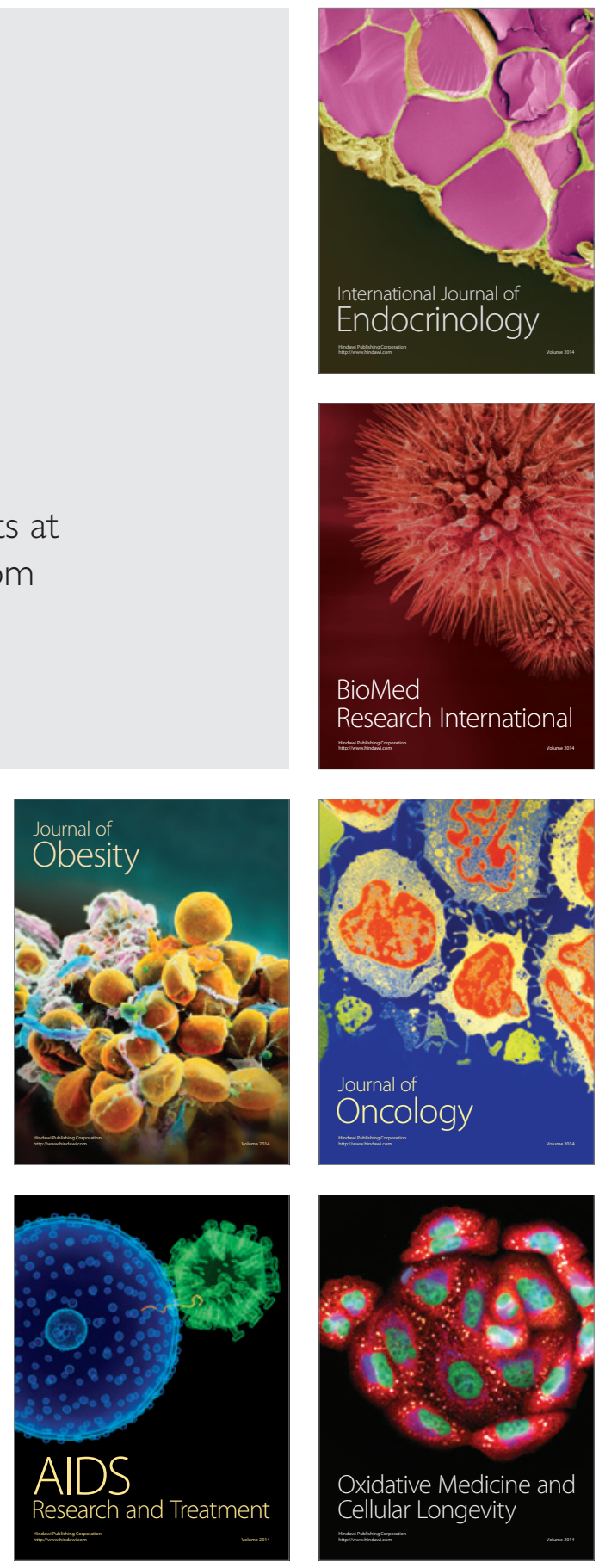